underlying periodic behaviour, with somewhat discrepant results. Of a total until now of seven investigations, only two teams found evidence of periodic behaviour; one group reported a transient pulse with a period of $20 \mathrm{~s}$ in both the optical and $\mathrm{X}$-ray region; the other group suggested the detection of pulses in the optical with periods of 117 and $182 \mathrm{~s}$ coexisting.

The existence of pulses in Sco X-1 has therefore been a matter of some controversy. But the latest observations of Frohlich cannot be lightly dismissed. On four successive days in June 1972, cvidence was found for the presence of low amplitude optical pulses with a period of $121 \mathrm{~s}$. This agrees quite woll with at least one of the periods found by an earlier group (P. A. Peldman et al., Nature, 225, 1123; 1970). A further fact, which if confirmed might help in the interpretation of these pulses, was a correlation between the brightness of the source and the period observed. On one night the overall output declined by a factor two in half an hour. This was associated with a decrease in the pulse period from 121 s to 118 s. Although Frohlich does not speculate as to the origin of these weak, transient pulses or this apparent brightness period correlation, a whole range of questions and possibilities immediately come to mind.

The sort of behaviour reported in Sco X-1 is remarkably similar to that of another class of binary system, the so-called dwarf novae. These show similar weak transient pulses which change in their period as the brightness changes. The dwarf novae are distinguished as a class by their explosive outbursts, brightening suddenly by several orders of magnitude and then more slowly declining back to a quiescent state. The outbursts recur at irregular, but approximately monthly intervals. The origin of these outbursts and of the underlying transient pulses remains a question of debate. But we might hope that the observation of somewhat similar pulse behaviour in Sco X-1 may help to elucidate the phenomena occuring in both types of system.

Two main lines of approach to account for transient pulses have so far been suggested: either oscillations, similar in some ways to the oscillations of the well known Cepheid pulsating stars; or rotation, as in the pulsars and stable pulsing X-ray sources mentioned earlier. Thus the choice seems to lie between what might be called swings and roundabouts. Both have been advanced to explain the pulses in dwarf novae. Put simply the swings model accounts for the pulses as being due to oscillations of the envelope of the accreting compact component, the pulses being excited by the nova outbursts themselves which are ascribed to phases of unstable nuclear burning on the accreting white dwarf. The roundabout model ascribes the outbursts to the sudden dumping of matter onto the dwarf as its companion undergoes phases of unstable mass outflow. It is well known that as a consequence of its angular momentum this accreting material must spiral slowly in onto the white dwarf rather than falling straight onto the surface. It is suggested that it, is just irregularities in this inward spiralling disk that gives rise to the pulse phenomenon.

It will be interesting to see how the roundabouts and swings fare in explaining in their rival fashions the behaviour of Sco X-1. Perhaps one shall then be able to get an answer to the truth or falsity of that old adage about gaining on swings and losing on roundabouts.

\section{Radiation of life in the Precambrian}

from our Palaeontology Correspondent

THe cause of the sudden explosive radiation of multicellular organisms near the beginning of the Cambrian period, after a long history of slow uniccllular evolution, is widely regarded as the foremost unsolved problem in palaeontology. In recent years two rather different approaches have been adopted in an attempt to solve the problem: the first emphasised external physical controls such as the changing level of atmospheric oxygen, whereas the second concentrated on biological aspects, such as the advent of eukaryotic cell organisation, sexuality or the formation of preservable skeletons. Neither approach, however, has been gencrally accepted.

In an important review, Schopf et al. (J. Paleont., 47, 1; 1973) point out that the evidence of Precambrian fossils suggests that eukaryotic organisms arose more than 1,300 and sexuality at least: 900 million years ago. The advent of sexuality should greatly have increased genetic variability and hence rates of evolutionary diversification, but the late Precambrian floras seem to be very impoverished in comparison with their living equivalents. The authors suggest that the long time gap, of $250-300$ million years, between the origins of sexuality and multicellular body organisation might have been a consequence of the gradual nature of the trend from a haploid to a diploid-dominated life cycle. This explanation does not seem very convincing, because an important evolutionary innovation such as diploid cell division should have risen to dominance very quickly. Another explanation of the rapid radiation of metaphytes and metazouns has been put forward by Stanley (Proc. natn. Acad. Sci. U.S.A., $70,1486 ; 1973)$, who applies a newly established 'cropping' principle to the problem.

Ecologists have acquired a substantial body of evidence that the addition of a trophic level to a given food web tends to promote inereased diversity at the next lower trophic level. Thus the removal of the chief natural carnivore from a community can lead to a drastic reduction in herbivore diversity as one or a few superior competitors among the latter achieve dominance. According to Stanley, the Precambrian trend from simple autotrophic producer to producer-herbivore and producer-herbivore-carnivore communities can be viewed as a long-term natural cropping experiment comparable to those performed by ecologists.

Initially an all-producer Precambrian organic world of bacteria and blue-green algae was characterised by low diversity and low rates of speciation. The system was self-limiting insofar as the changes needed to produce unicellular heterotrophs and metazoans had to arise slowly because diversification was slow. When cell-eating heterotrophs first arose in the late Precambrian they may have fed on bacteria. The adaptive breakthrough to feeding on algae led to the addition of successive trophic levels to the ecosystem. In general, diversification at any trophic level tends to promote diversification of the subjacent level by cropping and diversification of the suprajacent level by providing more feeding options. Thus a self-propagating mutual feedback system can arise. Stanley sees the explosive evolution of multicellular organisms as an inevitable consequence of the advent of heterotrophy. Supporting evidence is sought from the record of ancient stromatolites, which are laminated sedimentary structures produced by blue-green algal mats that trap and bind sediments. Their rapid decline at the close of the Precambrian has been considered to be the result of destruction of algal mats by animal grazers and burrowers, so that subsequently blue-green algae have only flourished in restricted environments, hostile to other organisms, such as supratidal flats. This suggests that blue-green algae and other photosynthetic autotrophs saturated aquatic environments until very late in the Precambrian.

It is obviously too carly to assess what chance Stanley's theory has of being more popular than its predecessors. It does seem, however, to have several definite strengths: it accounts for a wide variety of facts, it is basically simple and is purely biological, with no ad hoc invocation of external physical controls. 